University of Wollongong

Research Online

Faculty of Science, Medicine and Health -

Papers: Part B

Faculty of Science, Medicine and Health

$1-1-2020$

\title{
Carotenoid supplementation affects the post-hibernation performance of southern corroboree frogs
}

\author{
Emma Mclnerney \\ University of Wollongong, emcinern@uow.edu.au
}

Aimee J. Silla

University of Wollongong, asilla@uow.edu.au

Phillip G. Byrne

University of Wollongong, pbyrne@uow.edu.au

Follow this and additional works at: https://ro.uow.edu.au/smhpapers1

\section{Publication Details Citation}

Mclnerney, E., Silla, A. J., \& Byrne, P. G. (2020). Carotenoid supplementation affects the post-hibernation performance of southern corroboree frogs. Faculty of Science, Medicine and Health - Papers: Part B.

Retrieved from https://ro.uow.edu.au/smhpapers1/1266 


\title{
Carotenoid supplementation affects the post-hibernation performance of southern corroboree frogs
}

\begin{abstract}
(C) 2020 Copyright 2020 by Koninklijke Brill NV, Leiden, The Netherlands. Many animals hibernate to survive winter conditions, however, arousal from hibernation generates reactive oxygen species (ROS) that can cause oxidative stress. Dietary antioxidants, like carotenoids, may reduce oxidative stress during arousal from hibernation, and assist with post-hibernation recovery and performance. We tested the effect of carotenoid supplementation on exercise performance (escape-response and activity) in southern corroboree frogs (Pseudophryne corroboree) following initial arousal from hibernation (24-48 $\mathrm{h}$ post-arousal) and post-recovery (six weeks post-hibernation). Carotenoids did not affect performance following initial arousal. However, carotenoids improved escape-response six weeks post-hibernation, with carotenoid-supplemented frogs hopping faster and further in their first hop than unsupplemented frogs. Carotenoids also affected post-recovery activity, with carotenoid-supplemented frogs being less mobile than unsupplemented frogs. Carotenoids may affect post-hibernation performance by reducing oxidative stress or by increasing diet quality. Our study provides novel evidence for an effect of carotenoids on performance post-hibernation and highlights the importance of nutrition to hibernating organisms.
\end{abstract}

\section{Publication Details}

Mclnerney, E., Silla, A. \& Byrne, P. (2020). Carotenoid supplementation affects the post-hibernation performance of southern corroboree frogs. Behaviour, 157 (2), 121-142. 
1 Carotenoid supplementation affects the post-hibernation performance of southern

2 corroboree frogs

3

4 Emma P. McInerney ${ }^{1 *}$, Aimee J. Silla ${ }^{1} \&$ Phillip G. Byrne ${ }^{1}$

5

$6 \quad{ }^{1}$ School of Earth, Atmospheric and Life Sciences,

7 University of Wollongong,

8 Wollongong, NSW, 2522,

9 Australia

10

11 *Corresponding author email: epm509@uowmail.edu.au

$12 \mathrm{~T}+61242981932$

$13 \quad F+61242214135$

14

15 Word count: 5912

16 Figures: 3

17 Supplementary movies: 2

18 Declarations of interest: none

19

20 


\section{Summary}

23 Many animals hibernate to survive winter conditions, however, arousal from hibernation

24 generates reactive oxygen species (ROS) that can cause oxidative stress. Dietary antioxidants,

25 like carotenoids, may reduce oxidative stress during arousal from hibernation, and assist with

26 post-hibernation recovery and performance. We tested the effect of carotenoid

supplementation on exercise performance (escape-response and activity) in southern

corroboree frogs (Pseudophryne corroboree) following initial arousal from hibernation (24-

$48 \mathrm{~h}$ post-arousal) and post-recovery (six weeks post-hibernation). Carotenoids did not affect

30 performance following initial arousal. However, carotenoids improved escape-response six

31 weeks post-recovery, with carotenoid-supplemented frogs hopping faster and further in their

32 first hop than unsupplemented frogs. Carotenoids also affected post-recovery activity, with carotenoid-supplemented frogs being less mobile than unsupplemented frogs. Carotenoids may affect post-hibernation performance by reducing oxidative stress or by increasing diet quality. Our study provides novel evidence for an effect of carotenoids on performance posthibernation and highlights the importance of nutrition to hibernating organisms.

Keywords: anuran, activity, antioxidants, carotenoids, escape-response, hibernation 


\section{Introduction}

Hibernation is a strategy adopted by many animals to enable them to endure periods of extremely low winter temperatures (St-Pierre \& Boutilier, 2001; Bagnyukova et al., 2003).

During hibernation, individuals supress their metabolic rate, oxygen consumption, and activity, allowing them to survive extended periods without feeding (St-Pierre \& Boutilier, 2001). While hibernation is essential for the survival of many species, arousal from hibernation can cause significant physiological stress (Hermes-Lima et al., 2015). High oxygen intake needed to return to a normal metabolic rate, combined with increased energetic expense required to cope with elevated post-hibernation temperatures, generates a large amount of reactive oxygen species (ROS) that can harm the body (Bagnyukova et al., 2003; Hermes-Lima et al., 2015). ROS are radical molecules produced as a by-product of mitochondrial respiration which can oxidise otherwise stable cellular molecules (Powers et al., 2004). When the rate of ROS production exceeds the rate of ROS detoxification, the result is oxidative stress, which can cause irreversible damage to lipids, proteins, DNA, and organismal performance (Powers et al., 2004). During arousal from hibernation, an individual's antioxidant system (which includes both endogenous and exogenous antioxidants) functions to reduce oxidative damage (Hermes-Lima et al., 2015). While it is widely acknowledged that most hibernating animals have evolved the ability to increase endogenous antioxidant levels during arousal from hibernation (termed 'preparation for oxidative stress') (Hermes-Lima et al., 2015), many still experience higher than normal rates of oxidative stress (Bagnyukova et al., 2003; Niu et al., 2018).

A diet rich in exogenous antioxidants, received prior to hibernation, may improve the antioxidant systems capacity to lessen the effects of ROS-induced damage following hibernation. One group of exogenous antioxidants obtained by animals through their diet are 
carotenoids, a group of over 750 hydrocarbon compounds synthesised by photosynthetic plants, bacteria, and fungi (Svensson \& Wong, 2011). Dietary carotenoids are consumed by a diversity of animals and may strongly support post-hibernation exercise performance, encompassing traits such as escape-response and general activity, which are known to directly impact survival (Edmunds, 1974). Post-hibernation escape-response behaviour and activity are expected to dictate how likely an individual is to find resources (such as food and refuge) and escape predation following arousal. However, these behaviours can be energetically costly and elevate the production of ROS (Powers et al., 2004; Blount \& Matheson, 2006; Rowe et al., 2015). Increased consumption of carotenoids prior to hibernation is expected to reduce the stress experienced by individuals when exhibiting these behaviours post-hibernation, and, in turn, improve performance (Powers et al., 2004; Blount \& Matheson, 2006).

Beyond performance benefits received during arousal form hibernation, consumption of carotenoids may also be critically important during recovery from hibernation. Several studies have shown that ROS-induced stress can be experienced for several months following hibernation (Cooper et al., 1992; Feidantsis et al., 2012; Feidantsis et al., 2013; Hoelzl et al., 2016). Furthermore, individuals emerging from hibernation typically do so in spring, a season associated with rapid somatic growth, and increased investment in reproductive traits and processes; both of which are energetically costly activities known to generate high amounts of ROS (Blount, 2004; Metcalfe \& Alonso-Alvarez, 2010). Therefore, if damaging effects of ROS are cumulative (as suggested by Bagnyukova et al., 2003; Metcalfe \& Alonso-Alvarez, 2010), dietary antioxidants may become increasingly important in the weeks following arousal. While past studies have shown that exogenous antioxidants can improve performance (Aoi et al., 2003; Blount \& Matheson, 2006; Larcombe et al., 2008; Silla et al., 
2016), no study to date has investigated how dietary antioxidants influence performance following initial arousal from hibernation and following recovery from hibernation.

Anuran amphibians (frogs and toads) present excellent opportunities to test the effects of carotenoid supplementation on post-hibernation performance. Globally, a diversity of anuran species utilise hibernation as an overwintering survival strategy, and the effects of hibernation on antioxidant defences and oxidative stress in anurans has been well documented (Boutilier, 2001; St-Pierre \& Boutilier, 2001; Bagnyukova et al., 2003; Feidantsis et al., 2012; Prokic et al., 2017; Niu et al., 2018). Specifically, it has been shown that hibernating anurans can experience higher antioxidant activity than normal both during, and post-hibernation (Bagnyukova et al., 2003), but also that hibernation can increase levels of oxidative stress in heart and muscle tissue (Niu et al., 2018), and reduce muscle performance (Hudson and Franklin, 2002). At present, there is a lack of information regarding the long-term effects of hibernation on anuran performance, however two studies in water frogs (Pelophylax ridinbundus) have shown that hibernation can increase the long-term occurrence of heat shock proteins and oxidative stress, which is likely to have direct effects on performance (Feidantsis et al., 2012; Feidantsis et al., 2013).

The present study aimed to investigate the effect of dietary carotenoids on the posthibernation escape-response performance and general activity of southern corroboree frogs (Pseudophryne corroboree). P. corroboree enter hibernation during the onset of austral winter and evade freezing by burrowing into underground refuges (at the base of tussock grasses, under logs, and in sphagnum bogs) (Osborne, 1991; McFadden, 2019, personal comms). Past studies with $P$. corroboree have shown that dietary carotenoids can improve escape-response performance (Silla et al., 2016; McInerney et al., 2017), and modify exploratory behaviour (Kelleher, et al., 2019). In the present study, we tested the effect of carotenoid supplementation on the post-hibernation performance of adult $P$. corroboree 
120 following initial arousal from hibernation (24-48 hrs post-arousal) and following recovery

121 from hibernation (six weeks post-arousal) to explore whether exogenous antioxidants play a

122 role in post-hibernation performance. We predicted that dietary carotenoids would assist with

123 both initial arousal and recovery from hibernation, such that carotenoid-supplemented

124 individuals would perform significantly better than unsupplemented individuals at both time

125 periods. Further to this, we predicted that the performance of carotenoid supplemented, but

126 not unsupplemented, frogs would improve between initial arousal and recovery from

127 hibernation, reflecting the capacity for carotenoids to aid in post-hibernation recovery over 128 time.

\section{2. Materials and Methods}

131

\subsection{Study species}

133

134 Pseudophryne corroboree is a small (25-30 mm, snout-vent length) anuran (family:

135 Myobatrachidae) endemic to the Snowy Mountain region of New South Wales (Osborne, 136 1991). In this region, adult frogs enter a state of hibernation during the winter months

137 (Osborne, 1991). P. corroboree is characterised by bright yellow and black markings

138 (Osborne, 1991). As with other Pseudophryne species, their predominant mode of locomotion

139 is a slow to rapid crawl, although individuals hop short distances when threatened (Colefax,

140 1956; Silla et al., 2016). P. corroboree feed on algae during the larval life-stage (Osborne,

141 1991), and invertebrates during the adult life-stage (Osborne, 1991), both of which contain

142 carotenoids (Lichtenthaler, 1987). 
147 To test the effects of carotenoid supplementation on the post-hibernation escape-response

148 performance and general activity of $P$. corroboree, frogs were assigned to one of two dietary

149 treatments from hatching $(n=24$ per treatment). These were; $(1)$ an unsupplemented diet

150 containing no added carotenoids, and (2) a carotenoid-supplemented diet, which contained

151 added carotenoids from a broad-spectrum carotenoid mix (and potentially other plant-derived

152 nutrients; Superpig; Rapashy®, CA, USA). To quantify the effect of carotenoid

153 supplementation on escape-response and general activity following hibernation, behavioural

154 assays were conducted over two hibernation cycles that took place annually. Escape-response

155 assays were conducted between November 19, 2015 and January 1, 2016, and activity assays

156 were conducted in the following annual hibernation cycle between November 24, 2016 and

157 January 6, 2017. Details of these behavioural assays can be found in sections 2.5 and 2.6.

\subsection{Husbandry and nutrition prior to hibernation}

160

161 Fertilised $P$. corroboree eggs $(\mathrm{n}=48)$ used in this study were obtained from a captive colony held at Melbourne Zoo, Australia. Eggs were transported to the University of Wollongong on July 19, 2013 and stimulated to hatch via flooding with reverse-osmosis (R.O.) water. During

164 both larval (tadpole) and adult (post-metamorphic) life-stages, animals were housed

165 individually according to husbandry methods stated elsewhere (McInerney et al., 2016; Silla et al., 2016). At the commencement of the experimental period, tadpoles were randomly assigned to either an unsupplemented dietary treatment or a carotenoid-supplemented dietary treatment $(\mathrm{N}=24$ frogs per treatment). The unsupplemented tadpole dietary treatment 
$17020 \mathrm{ml}$ of R.O. water, drawn into syringes, frozen and then thawed prior to use. For the first

171 two months of the experimental period tadpoles received two droplets (range $=0.015 \mathrm{~g}-$

$1720.018 \mathrm{~g}$ dry mass) of homogenised food suspension three times a week. Food supply was then

173 increased to four droplets (range $=0.030 \mathrm{~g}-0.036 \mathrm{~g}$ dry mass) until metamorphosis

174 (forelimb emergence: Gosner stage 42; Gosner, 1960). The unsupplemented tadpole dietary

175 treatment contained low levels of total carotenoids $\left(0.015 \mathrm{mg} \mathrm{g}^{-1}\right)$ (see Silla et al., 2016).

176 During the tadpole life-stage, the carotenoid-supplemented dietary treatment consisted of the

177 unsupplemented dietary treatment described above, supplemented with $0.040 \mathrm{~g}$ of carotenoid

178 mixture containing 20 different carotenoid pigments (Superpig; Rapashy®, CA, USA) (Silla

179 et al., 2016).

180 During metamorphosis (Gosner stage 42 to 46), individuals were not provided with food as

181 nutritional needs are met by re-absorption of the tail. Upon completion of metamorphosis,

182 frogs received live crickets twice weekly so that food was available ad libitum. Frogs in the

183 unsupplemented adult dietary treatment were provided with crickets that had been fed $48 \mathrm{~h}$

184 earlier with granny-smith apple (poor in carotenoids). The unsupplemented adult diet

185 contained very small amounts of total carotenoids $\left(0.005 \mathrm{mg} \mathrm{g}^{-1}\right)$. Frogs in the carotenoid-

186 supplemented dietary treatment were provided with crickets that had been fed $48 \mathrm{~h}$ earlier

187 with carrot (rich in carotenoids) and dusted with $1 \mathrm{~g}$ of carotenoid mixture (Superpig;

188 Rapashy®, CA, USA). In total, the carotenoid-supplemented adult dietary treatment

189 contained $1.152 \mathrm{mg} \mathrm{g}^{-1}$ of carotenoids (see Silla et al., 2016). Once a week, crickets in both

190 dietary treatments were dusted with $0.200 \mathrm{~g}$ of calcium powder (Repti-Cal, Aristopet,

191 Australia) prior to feeding to prevent nutrient deficiencies. Crickets ranged in age from $2-10$

192 days old for both dietary treatments. Frogs received experimental diets for approximately two

193 and a half years prior to escape-response assays being conducted, and three and a half years

194 prior to activity trials being conducted (see details below). 
197 To simulate the onset of winter and induce frogs to enter a state of hibernation, the

198 temperature in the experimental room was dropped progressively from $20^{\circ} \mathrm{C}$ to $10^{\circ} \mathrm{C}$ (Figure

199 1). In preparation for hibernation, once the temperature was reduced to $13^{\circ} \mathrm{C}$, feeding ceased

200 and frogs were not fed until arousal from hibernation (a total of six weeks; Figure 1).

201 Following the first week of fasting, the temperature was reduced to $10^{\circ} \mathrm{C}$. At this temperature,

202 frogs entered a state of hibernation and displayed very little activity. During this time, the

203 lighting within the experimental room was placed on an 11: $13 \mathrm{~h}$ light: dark cycle with a 15

204 min dusk period to simulate conditions experienced in Kosciusko National Park (in the

205 Snowy Mountains) during winter (June - August) (Bureau of Meteorology, 2015). Following

206 a four-week period at $10^{\circ} \mathrm{C}$, temperature was incrementally increased over a two-week period

207 to reach $15^{\circ} \mathrm{C}$, which caused frogs to arouse from hibernation. Initial arousal behavioural

208 assays took place $24-48 \mathrm{~h}$ after temperatures were increased to $15^{\circ} \mathrm{C}$ (hereafter referred to as

209 trial week 0) (Figure 1). The following day, feeding resumed and frogs were fed their adult

210 dietary treatments for a six-week recovery period before being exposed to a second round of

211 behavioural assays (hereafter referred to as trial week 6) (Figure 1).

212 


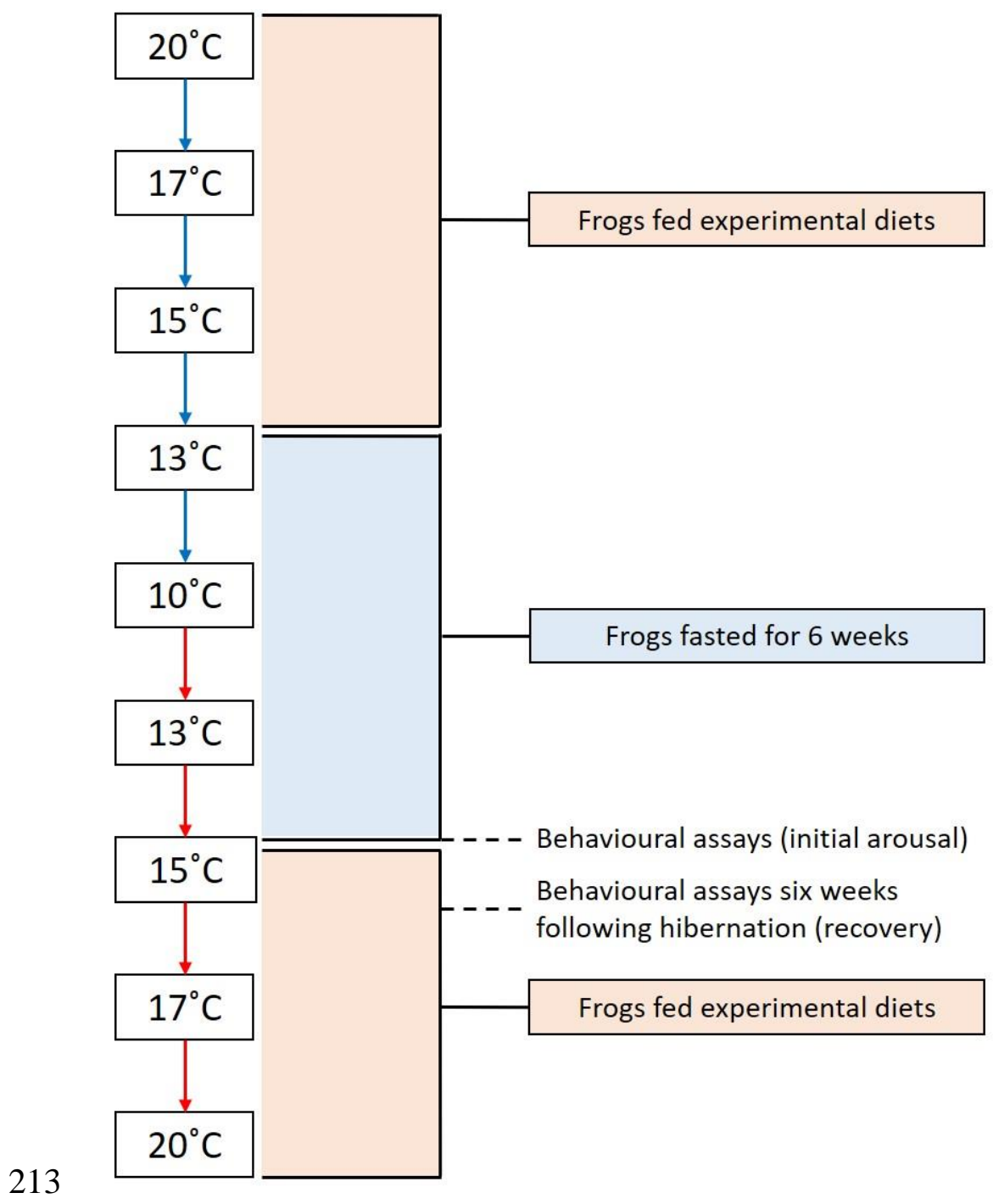

214 Figure 1. Yearly hibernation cycle for P. corroboree. Frogs were unfed at $13^{\circ} \mathrm{C}$ and entered a

215 state of hibernation at $10^{\circ} \mathrm{C}$. Frogs were aroused from hibernation as temperatures returned to

$21615^{\circ} \mathrm{C}$. All behavioural assays were conducted at $15^{\circ} \mathrm{C}$ following hibernation. Initial arousal

217 behavioural assays were conducted $24-48 \mathrm{~h}$ following arousal from hibernation when frogs

218 were still fasted. Recovery behavioural assays were conducted six weeks following

219 hibernation when frogs had resumed their experimental diets. 
226 Escape performance trials were conducted on November 19 and 20, 2015 (trial week 0) and

227 on December 31, 2015 and January 1, 2016 (trial week 6). In order to quantify escape-

228 response, frogs were subjected to hopping performance trials where individuals were pursued

229 to the end of a narrow hopping track $(30 \mathrm{~cm} \mathrm{x} 4 \mathrm{~cm} \mathrm{x} 4 \mathrm{~cm})$ by a model Alpine Copperhead

230 snake (Austrelaps ramsayi), found naturally in the same range as $P$. corroboree. Before trials

231 commenced, frogs were placed in front of the model snake, separated by an opaque divider

232 where they were held by the researcher until they remained motionless. Frogs were

233 approximately $5 \mathrm{~cm}$ from the model snake before trials began. The opaque divider was then

234 removed and the model snake moved towards the frog at a constant speed of $0.750 \mathrm{~cm} \mathrm{~s}^{-1}$ via an automated motorised pulley system (Tamiya Corp., Aliso Viejo, CA, USA) controlled by

a 12 V 36 RPM motor (Soanar, Sydney, NSW, Australia) attached to a 12 V DC speed controller and Plus Switch mode regulated plug pack (Powertech, Taiwan, China) (Supplementary movie S1). Between trials, the hopping track was wiped clean with ethanol and then R.O. water to remove any chemosignals that may have influenced individuals' performance. An equal number of representatives from each dietary treatment were tested on each trial day, and trial order was randomised to avoid temporal effects. All trials were video recorded using a high definition Sony Exmor R Handycam mounted on a tripod approximately $50 \mathrm{~cm}$ above the hopping track. Videos were later viewed using Windows

244 Movie Maker software and the following behavioural response variables were quantified; (1)

245 hopping speed $\left(\mathrm{cm} \mathrm{s}^{-1}\right.$ ) (of the first hop), (2) length of the first hop (cm), and (3) total hopping distance $(\mathrm{cm})$ in response to the first tap from the snake. 
251 Activity assays were conducted on November 24 and 25, 2016 (trial week 0) and on January 2525 and 6, 2017 (trial week 6). In order to quantify general activity, frogs were moved from 253 their home containers into experimental containers $(31.9 \mathrm{~cm} \times 17.8 \mathrm{~cm} \times 20 \mathrm{~cm})$. Each 254 experimental container was lined on the bottom with white corflute, and the walls of the experimental container were lined with black opaque plastic to remove visual contact with neighbouring frogs. Frogs were acclimated to experimental containers under a black opaque plastic cup for five minutes. Following this acclimation period, the cup was removed using a pulley system and the activity behaviour of each individual was video recorded for 60 minutes (Supplementary movie S2). Trials were simultaneously completed in six blocks of eight individuals. Within each block, there were equal representatives from each dietary treatment and trial order was randomised to avoid temporal effects. During trials, the researcher was not present in the room in order to remove any observer effects on the frogs' activity. Between trials, the experimental containers were wiped clean with ethanol and then R.O. water to remove any chemosignals that may have influenced individuals' performance. All trials were video recorded using high definition Panasonic HC-W580M cameras suspended approximately $60 \mathrm{~cm}$ above the experimental containers. Videos were later rewatched in Anymaze software and the following response variables were quantified; (1) distance travelled (cm), (2) the number of mobile episodes, defined as the number of times

269 frogs became active during the activity assay, and (3) time mobile (s). Frogs were classified as being immobile after five seconds of inactivity. 
276 For each behavioural assay, neither body size (snout-vent length or mass) nor sex had a

277 significant influence on any of the response variables measured, and body size did not change

278 significantly during behavioural assay periods. Therefore, body size and sex were not

279 included as co-variates in any subsequent statistical analyses. For each behavioural assay,

280 four individuals were excluded from analyses due to deaths within the trial period or unusable

281 behavioural trials (therefore Unsupplemented dietary treatment; $n=21$, Carotenoid-

282 supplemented dietary treatment; $\mathrm{n}=23$ ). Because data were not normally distributed non-

283 parametric tests were used. To test the effect of dietary treatment on hopping speed, length of 284 the first hop, hopping distance, distance travelled, number of mobile episodes, and time mobile for each trial week (trial week 0 and trial week 6), we ran Wilcoxon rank sum tests. In each model, dietary treatment was the explanatory variable and either hopping speed, length of the first hop, hopping distance, distance travelled, number of mobile episodes, or time mobile was the response variable. To test the effect of trial week (time) on hopping speed, length of the first hop, hopping distance, distance travelled, number of mobile episodes, and time mobile for each dietary treatment, we ran Friedman's tests. In each model, trial week was the explanatory variable, individual ID was the block, and hopping speed, length of the first hop, hopping distance, distance travelled, number of mobile episodes, or time mobile was the response variable. All statistical analyses were performed in R studio 1.1.447. 
301 All procedures in this experiment were carried out following evaluation and approval from

302 the University of Wollongong's Animal Ethics Committee (approved number AE13/13)

303 following ASAB/ABS ARRIVE guidelines.

304

305

3. Results

306

307

\subsection{Escape-response assays}

308

\subsubsection{Hopping speed}

311 Dietary treatment had no significant effect on hopping speed in trial week 0 (mean \pm SEM;

312 Unsupplemented dietary treatment $=1.929 \pm 0.555 \mathrm{~cm} \mathrm{~s}^{-1}$, Carotenoid-supplemented dietary

313 treatment $\left.=1.956 \pm 0.444 \mathrm{~cm} \mathrm{~s}^{-1}\right)($ Wilcoxon: $W=257.500, P=0.709)($ Figure 2(A) $)$.

314 However, carotenoid-supplemented frogs hopped significantly faster than unsupplemented

315 frogs in trial week 6 (mean \pm SEM; Unsupplemented dietary treatment $=2.685 \pm 0.623 \mathrm{~cm} \mathrm{~s}^{-}$

316 , Carotenoid-supplemented dietary treatment $\left.=5.450 \pm 0.861 \mathrm{~cm} \mathrm{~s}^{-1}\right)($ Wilcoxon: $W=338, P$

$317=0.023)($ Figure 2(B)). Furthermore, individuals from the carotenoid-supplemented dietary

318 treatment hopped significantly faster in trial week 6 than in trial week 0 (Friedman's test; $x^{2} 1$

$319=10.714, P=0.001$ ), whereas individuals in the unsupplemented dietary treatment did not

320 change in their hopping speed between trial weeks (Friedman's test; $x^{2}{ }_{1}=0.053, P=0.819$ ).

321 These results indicate that individuals from the carotenoid-supplemented dietary treatment

322 improved in their hopping performance over the six week post-hibernation recovery period. 
326 Dietary treatment had no significant effect on length of the first hop in trial week 0 (mean \pm

327 SEM; Unsupplemented dietary treatment $=0.614 \pm 0.154 \mathrm{~cm}$, Carotenoid-supplemented

328 dietary treatment $=0.913 \pm 0.162 \mathrm{~cm})($ Wilcoxon: $W=288.500, P=0.259)$ (Figure 2@).

329 However, carotenoid-supplemented frogs hopped significantly further in their first hop than unsupplemented frogs in trial week $6($ mean \pm SEM; Unsupplemented dietary treatment $=$ $0.929 \pm 0.180 \mathrm{~cm}$, Carotenoid-supplemented dietary treatment $=1.652 \pm 0.249 \mathrm{~cm})$

(Wilcoxon: $W=333, P=0.029$ ) (Figure 2(D)). Furthermore, individuals from the carotenoidsupplemented dietary treatment hopped significantly further in their first hop in trial week 6 than in trial week 0 (Friedman's test; $x^{2}{ }_{1}=8.895, P=0.003$ ), whereas individuals in the unsupplemented dietary treatment did not change in their hopping speed between trial weeks (Friedman's test; $x^{2}{ }_{1}=0.529, P=0.467$ ).

\subsubsection{Hopping distance}

Dietary treatment had no significant effect on hopping distance in trial week 0 (mean \pm SEM;

341 Unsupplemented dietary treatment $=1.724 \pm 0.453 \mathrm{~cm}$, Carotenoid-supplemented dietary treatment $=1.022 \pm 0.19 \mathrm{~cm})($ Wilcoxon: $W=219.500, P=0.603)($ Figure $2(\mathrm{E}))$. While carotenoid-supplemented individuals hopped further than unsupplemented individuals in trial week 6 (mean \pm SEM; Unsupplemented dietary treatment $=2.881 \pm 0.100 \mathrm{~cm}$, Carotenoidsupplemented dietary treatment $=4.696 \pm 1.100 \mathrm{~cm})($ Figure $2(\mathrm{~F}))$, this was marginally unsignificant (Wilcoxon: $W=322, P=0.058$ ). Individuals from the carotenoid-supplemented

347 dietary treatment hopped significantly further in trial week 6 than in trial week 0 (Friedman's 348 test; $x^{2}{ }_{l}=13.762, P<0.001$ ), whereas individuals in the unsupplemented dietary treatment 
349 did not change in their hopping speed between trial weeks (Friedman's test; $x^{2}{ }_{1}=1.471, P=$ $350 \quad 0.225)$.

351
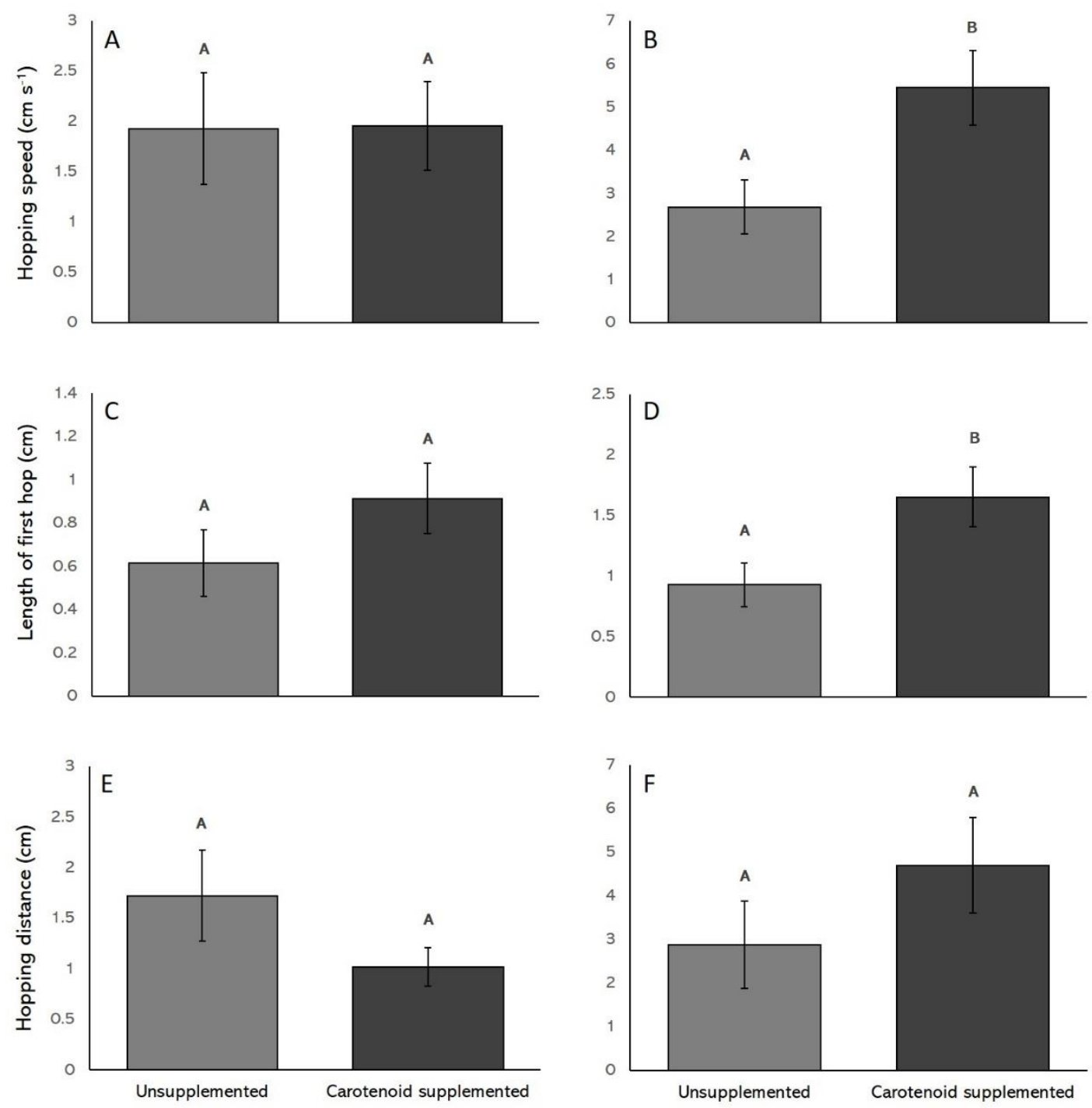

Trial week 0

Trial week 6

353 Figure 2. Effect of carotenoid supplementation on; hopping speed $\left(\mathrm{cm} \mathrm{s}^{-1}\right)$ in (A) trial week 0 354 and (B) trial week 6, length of the first hop $(\mathrm{cm})$ in $(\mathrm{C})$ trial week 0 and $(\mathrm{D})$ trial week 6 , and 355 hopping distance $(\mathrm{cm})$ in $(\mathrm{E})$ trial week 0 and $(\mathrm{F})$ trial week 6 for $P$. corroboree. Dietary

356 treatments were; Unsupplemented $(\mathrm{n}=21)$ or Carotenoid-supplemented $(\mathrm{n}=23)$. Data shown 357 are untransformed means \pm SEM. Columns not connected by a letter are significantly different. 


\subsubsection{Distance travelled}

362

363 Dietary treatment had no significant effect on the distance travelled in trial week 0 (mean \pm

364 SEM; Unsupplemented dietary treatment $=612.891 \pm 83.479 \mathrm{~cm}$, Carotenoid-supplemented dietary treatment $=655.739 \pm 106.375 \mathrm{~cm})($ Wilcoxon: $W=236, P=0.907)($ Figure $3(\mathrm{~A}))$, or trial week 6 (mean \pm SEM; Unsupplemented dietary treatment $=7.322 \pm 136.651 \mathrm{~cm}$,

Carotenoid-supplemented dietary treatment $=521.413 \pm 65.917 \mathrm{~cm})($ Wilcoxon: $W=192, P$ $=0.252)($ Figure 3(B)). Furthermore, there was no effect of trial week on the distance travelled by individuals in the carotenoid-supplemented dietary treatment (Friedman's test; $x^{2}{ }_{1}=0.043, P=0.835$ ), or the unsupplemented dietary treatment (Friedman's test; $x^{2}{ }_{1}=$ $0.048, P=0.827)$.

\subsubsection{Number of mobile episodes}

374

375

Dietary treatment had no significant effect on the number of mobile episodes in trial week 0 (mean \pm SEM; Unsupplemented dietary treatment $=99.238 \pm 7.979$, Carotenoidsupplemented dietary treatment $=104.478 \pm 9.142)($ Wilcoxon: $W=263.500, P=0.613)$ (Figure 3(C). However, unsupplemented frogs had significantly more mobile episodes than carotenoid-supplemented frogs in trial week 6 (mean \pm SEM; Unsupplemented dietary treatment $=94.143 \pm 10.171$, Carotenoid-supplemented dietary treatment $=59.826 \pm 5.005)$

381 (Wilcoxon: $W=98, P<0.001$ ) (Figure 3(D)). Furthermore, individuals from the carotenoidsupplemented dietary treatment had significantly fewer mobile episodes in trial week 6 than

383 in trial week 0 (Friedman's test; $x^{2}{ }_{1}=15.696, P<0.001$ ), whereas individuals in the 
unsupplemented dietary treatment did not change in the number of mobile episodes they exhibited between trial weeks (Friedman's test; $x^{2}{ }_{l}=0.048, P=0.827$ ).

386

\subsubsection{Time mobile}

388

389 Dietary treatment had no significant effect on the time mobile in trial week 0 (mean \pm SEM;

390 Unsupplemented dietary treatment $=1128.719 \pm 129.525 \mathrm{~s}$, Carotenoid-supplemented dietary

391 treatment $=1086.191 \pm 115.390 \mathrm{~s})($ Wilcoxon: $W=237, P=0.926)($ Figure $3(\mathrm{E}))$, or trial

392 week 6 (mean \pm SEM; Unsupplemented dietary treatment $=1215.743 \pm 187.797 \mathrm{~s}$,

393 Carotenoid-supplemented dietary treatment $=899.809 \pm 107.607$ s) $($ Wilcoxon: $W=187, P=$

394 0.207) (Figure 3(F)). Furthermore, there was no effect of trial week on the time mobile for

395 individuals in the carotenoid-supplemented dietary treatment (Friedman's test; $x^{2}{ }_{1}=0.043, P$

$396=0.835$ ), or the unsupplemented dietary treatment (Friedman's test; $x^{2}{ }_{1}=0.048, P=0.827$ ). 

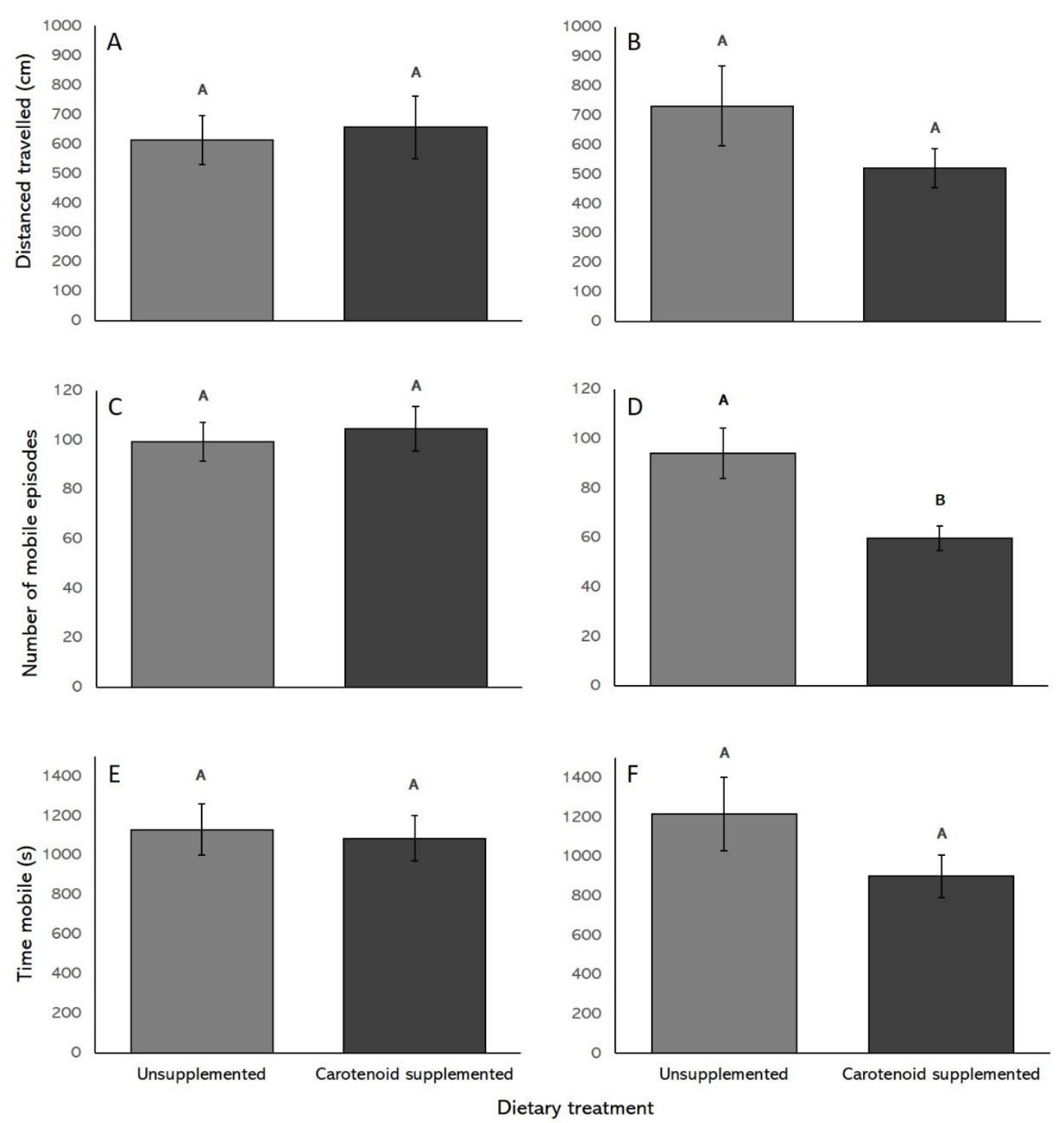

Trial week 0

Trial week 6

399 Figure 3. Effect of carotenoid supplementation on the; distance travelled in (A) trial week 0 400 and (B) trial week 6, number of mobile episodes in (C) trial week 0 and (D) trial week 6, and 401 time mobile (s) in (E) trial week 0 and (F) trial week 6 for $P$. corroboree. Dietary treatments 402 were; Unsupplemented $(n=21)$ or Carotenoid-supplemented $(n=23)$. Data shown are 403 untransformed means \pm SEM. Columns not connected by a letter are significantly different. 
410 While hibernation is essential for the survival of many organisms, high levels of reactive

411 oxygen species (ROS), generated during arousal from hibernation, are expected to negatively

412 impact post-hibernation performance. Dietary supplementation with exogenous antioxidants,

413 such as carotenoids, is expected to limit the degree of oxidative stress experienced during

414 arousal from hibernation, and aid in post-hibernation recovery and performance. Both escape-

415 response and general activity are critical for the survival of individuals following hibernation

416 but can cause significant oxidative stress. In this study, we tested the effect of dietary

417 carotenoids on the post-hibernation escape-response performance and general activity of the

418 southern corroboree frog, Pseudophryne corroboree. Individuals were fed either a

419 carotenoid-supplemented or unsupplemented dietary treatment and their escape-response and

420 general activity were assayed following initial arousal from hibernation (24-48 hrs post-

421 arousal; trial week 0 ) and following recovery from hibernation (six weeks post-arousal; trial

422 week 6). In regard to escape-response, carotenoid supplementation had no effect on any

423 measure of performance (hopping speed, length of the first hop, or hopping distance) in trial

424 week 0 . However, the escape-response performance of carotenoid-supplemented individuals

425 significantly improved over time, while the performance of unsupplemented individuals

426 remained unchanged. Overall, by trial week 6, carotenoid-supplemented individuals

427 outperformed unsupplemented individuals with regard to their hopping speed, and length of

428 the first hop, but not hopping distance. In regard to activity, there was no significant

429 difference in performance between dietary treatments in trial week 0 (distance travelled,

430 number of mobile episodes, or time mobile). However, by trial week 6, carotenoid-

431 supplemented individuals had fewer mobile episodes than unsupplemented individuals during

432 trials and had significantly fewer mobile episodes over time. These results indicate that 
carotenoid supplementation did not improve performance following initial arousal from

434 hibernation, but that dietary carotenoids enhanced escape-response performance and significantly influenced activity following recovery from hibernation. supplementation on either the escape-response performance or general activity of frogs following arousal from hibernation (trial week 0). One reason for this might be that the carotenoid stores of frogs were depleted during hibernation. While hibernation involves severe metabolic depression to reduce the likelihood of starvation, nutrient stores within the body can still be metabolised (Boutilier, 2001). For instance, European common frogs, Rana temporaria, only supress their metabolic rate by $50 \%$ during hibernation, and when exposed to more than three weeks of hibernation, rapidly consume their glycogen and carbohydrate stores (Boutilier, 2001). It is not known whether frogs deplete carotenoid stores during hibernation, but this outcome has been reported in other ectotherms that undergo estivation. For example, Helisoma trivolvis snails have been found to have a significant reduction in their lutein store during periods of estivation (metabolic depression during extended dry periods) (Arthur et al., 2006). An alternative explanation for our results is that the generation of ROS during arousal from hibernation may have been too great for the carotenoid dose administered to have a beneficial effect. Arousal from hibernation is a physiologically challenging event that generates large amounts of ROS (Bagnyukova et al., 2003; Niu et al., 2018). When ROS production exceeds the capacity of the antioxidants system to quench and stabilise it, oxidative stress results (Powers et al., 2004). Previously, arousal from hibernation has been shown to cause oxidative damage to lipids, heart tissue, and gastrocnemius muscle tissue in anuran amphibians (Bagnyukova et al., 2003; Niu et al., 2018). Therefore, it is

456 possible that frogs in our study experienced similar damaging affects following arousal from 457 hibernation. 

escape-response performance or general activity in trial week 0 may be that carotenoids were not taken up or assimilated prior to hibernation. However, this seems highly unlikely because

461 frogs in the present study received carotenoids for several years prior to testing. Moreover, a

462 previous study with these animals demonstrated that carotenoid supplementation improves

463 escape-response performance prior to hibernation (Silla et al., 2016). Therefore, an

464 alternative, and more plausible, explanation may be that supplementation with carotenoids limited the upregulation of endogenous antioxidants. During times of stress, ROS generation can trigger the upregulation of the endogenous antioxidants system, which helps to reduce

467 oxidative damage (Oztasan et al., 2004; Peternelj \& Coombes, 2011). In preparation for the 468 overproduction of ROS associated with arousal from hibernation, many species have evolved the ability to produce high levels of endogenous antioxidants (deemed 'preparation for stress') (Hermes-Lima et al., 1998; Hermes-Lima et al., 2015). However, it has been suggested that supplementation with exogenous antioxidants can limit the performance of the endogenous antioxidant system and, in some cases, even result in a greater incidence of 473 oxidative damage (Peternelj \& Coombes, 2011). While this theory remains to be tested during arousal from hibernation, it would explain why the escape-response performance of carotenoid-supplemented individuals did not differ from unsupplemented individuals immediately after arousal. To conclusively determine the effect of ROS, endogenous

477 antioxidants, and dietary carotenoids on post-hibernation performance, circulating

478 antioxidants and markers of oxidative stress in target tissue and blood would need to be 479 quantified. This was not possible in the present study due to the critically endangered status of $P$. corroboree. However, conducting this work in other less vulnerable overwintering anurans would provide important insights into the relative roles of the endogenous and 482 exogenous antioxidants in mitigating oxidative stress prior to, and following, hibernation. 

over the recovery period and outperformed unsupplemented individuals in trial week 6 (when considering hopping speed, and length of the first hop). This finding supports our hypothesis that carotenoids may act as antioxidants during recovery from hibernation, and during escape-response episodes following recovery. Arousal from hibernation can generate significant ROS and result in oxidative stress that can have lasting negative effects

492 (Feidantsis et al., 2012; Feidantsis et al., 2013). As antioxidants, carotenoids might help to 493 reduce ROS-induced stress experienced during recovery from hibernation, resulting in better performance. To date, only two studies have directly measured long-term cell and DNA damage caused by hibernation, but both have found that negative effects of hibernation can last for several months (Feidantsis et al., 2013; Hoelzl et al., 2016). To conclusively

497 determine whether carotenoids act as antioxidants to reduce cell damage during recovery 498 from hibernation, future studies would benefit from measuring ROS generation, oxidative stress, and circulating carotenoid levels.

500 Until such work is conducted, it is possible that there might be alternative explanations for

501 the carotenoid-mediated benefit to performance we have reported. One possibility is that carotenoids help to improve the general nutritional quality of the diet (Ogilvy \& Preziosi, 2012), which may directly affect the recovery of individuals. Specifically, carotenoid

504 supplementation may allow more resources, such as lipids and proteins, to be allocated to 505 bodily functions that improve the condition of individuals following hibernation- a time 506 typically associated with rapid somatic growth and preparation for reproduction. Another possibility is that the positive effect of supplementation was linked to the presence of other 
508 beneficial compounds in the supplement, as this was a plant extract mix containing several unknown compounds. To eliminate any potential for effects causes by unknown performance

510 enhancers, we recommend that future studies supplement diets with pure carotenoids.

511 Results from the activity behavioural assay showed that the activity of carotenoid-

512 supplemented individuals decreased over the post-hibernation recovery period (when

513 considering number of mobile episodes). By contrast, unsupplemented individuals did not

514 differ in their activity between trial week 0 and trial week 6 . As a result, the number of

515 mobile episodes was significantly lower in carotenoid-supplemented individuals compared to

516 unsupplemented individuals in trial week 6 . This result was unexpected, because, due to their

517 antioxidant properties, carotenoids were predicted to increase the post-hibernation activity

518 levels of frogs. Unsupplemented individuals may have exhibited more mobile episodes than

519 carotenoid-supplemented individuals following post-hibernation recovery because they

520 needed to increase their foraging intensity to satiate their elevated metabolic requirements

521 (Weimerskirch et al., 2003). It is believed that most animals will maximise foraging efficiency to invest in costly traits (e.g. somatic growth, development, ornamentation), while also conserving energy (Weimerskirch et al., 2003). However, when an individual's

524 nutritional state does not meet the requirements for maintaining costly traits, they may need

525 to increase their foraging activity (Weimerskirch et al., 2003). In the present study,

526 individuals fed carotenoid-supplemented diets may have had fewer mobile episodes in trial week 6 because they had access to a higher quality diet which met their metabolic demands during recovery. By comparison, unsupplemented individuals may have been more active as they needed to spend more time foraging to meet their metabolic demands. To determine

530 whether carotenoids improve foraging efficiency, future studies should quantify how carotenoid supplementation influences foraging dynamics. It would be particularly 
informative to investigate how different doses of carotenoids influence metabolic demands and how any associated physiological changes influence foraging efficiency.

More broadly, the findings of our study advance our understanding of the role of exogenous antioxidants in organismal functioning following hibernation. Past studies concerning the importance of antioxidants following hibernation have centred on understanding the role of endogenous antioxidants on post-hibernation stress. These studies have found that individuals can increase their endogenous antioxidant capacity to prepare for the stress of arousal from hibernation (see Hermes-Lima et al., 2015), but they may still experience some oxidative damage during arousal and recovery from hibernation (Bagnyukova et al., 2003; Feidantsis et al., 2013; Hoelzl et al., 2016; Niu et al., 2018). Our study is the first to demonstrate a positive influence of exogenous antioxidants on the

543 performance of individuals following hibernation, drawing attention to the possibility that

544 dietary antioxidants are more important to species that undergo hibernation than currently realised. Studies in various hibernating species are now needed to ascertain the general benefit of exogenous antioxidants to post-hibernation performance. Knowledge in this area will deepen our understanding of the links between nutrition and performance. supplementation on the post-hibernation performance of the southern corroboree frog, $P$. corroboree. We found no effect of carotenoid supplementation on the performance of frogs immediately following arousal from hibernation, however, carotenoid supplementation improved escape-response performance and significantly influenced activity six weeks postrecovery. Investigating the extent to which other species rely on dietary antioxidants to maintain post-hibernation performance will advance our understanding of the nutritional needs of hibernating organisms. 


\section{Supplementary material}

558

559

Supplementary material is available at Behaviour online.

560

561

Acknowledgements

562

563 The authors thank Shannon Kelleher for help with animal husbandry and conducting

564 behavioural assays. The authors thank staff in the Herpetofauna division at Zoos Victoria, in

565 particular Deon Gilbert for providing the P. corroboree eggs used in this study. We also

566 thank Michael McFadden and Adam Skidmore from Taronga Zoo for technical assistance

567 preparing our captive rearing facilities and advice on animal husbandry. We acknowledge the support of the New South Wales Department of Planning, Industry and Environment, particularly Dr David Hunter and members of the corroboree frog recovery team.

570

\section{$571 \quad$ Funding}

572

573 This work was supported by grants from the Australian Research Council [Linkage Grant 574 LP170100351] and NSW Environmental Trust [grant no. 2017/RD/0138] awarded to P.B. and A.S. in addition to the Holsworth Research Endowment - Equity Trustees Charitable

576 Foundation \& the Ecological Society of Australia awarded to E.M. The work was also

577 conducted while E.M. was in receipt of an Australian Government Research Training 578 Program (RTP) Scholarship.

579

580 
Aoi, W., Naito, Y., Sakuma, K., Kuchide, M., Tokuda, H., Maoka, T., Toyokuni, S., Oka, S.,

Yasuhara, M. \& Yoshikawa, T. (2003). Astaxanthin limits exercise-induced skeletal and cardiac muscle damage in mice. - Antioxid Redox Signal. 5: 139-144.

587

Arthur, B., Fried, B. \& Sherma, J. (2006). Effects of Estivation on Lutein and $\beta$-Carotene

Concentrations in Biomphalaria glabrata (NMRI Strain) and Helisoma trivolvis (Colorado

Strain) Snails as Determined by Quantitative High Performance Reversed Phase Thin Layer

Chromatography. - J Liq Chromatogr Relat Technol. 29: 2159-2165.

Bagnyukova, T. V., Storey, K. B. \& Lushchak, V. I. (2003). Induction of oxidative stress in

Rana ridibunda during recovery from winter hibernation. - J Thermal Biol. 28: 21-28.

593

Blount, J. D. (2004). Carotenoids and life-history evolution in animals. - Arch Biochem

Biophys. 430: 10-15.

Blount, J. D. \& Matheson, S. M. (2006). Effects of carotenoid supply on escape flight responses in zebra finches, Taeniopygia guttata. - Anim. Behav. 72: 595-601.

597 Boutilier, R. G. (2001). Mechanisms of metabolic defense against hypoxia in hibernating 598 frogs. - Respir Physiol. 128: 365-377.

Colefax, A. (1956). New information on the corroboree frog (Pseudophryne corroboree

600 Moore). - Proc. Linn. Soc. N.S.W. 80: 258-266.

601 Cooper, E. L., Wright, R. K., Klempau, A. E. \& Smith, C. T. (1992). Hibernation alters the 602 frog's immune system. - Cryobiology. 29: 616-631.

603 Edmunds M. (1974). Defence in Animals: A Survey of Anti-Predator Defences. Longman, 604 London.

605 Feidantsis, K., Anestis, A., Vasara, E., Kyriakopoulou-Sklavounou, P. \& Michaelidis, B. 606 (2012). Seasonal variations of cellular stress response in the heart and gastrocnemius muscle 
607 of the water frog (Pelophylax ridibundus). - Comp Biochem Physiol A Mol Integr Physiol. 608 162: 331-339.

609 Feidantsis, K., Anestis, A. \& Michaelidis, B. (2013). Seasonal variations of anti-/apoptotic 610 and antioxidant proteins in the heart and gastrocnemius muscle of the water frog Pelophylax 611 ridibundus. - Cryobiology. 67: 175-183.

612 Gosner, K. L. (1960). A simplified table for staging anuran embryos and larvae with notes on 613 identification. - Herpetologica. 16: 183-190.

614 Hermes-Lima, M., Storey, J. M. \& Storey, K. B. (1998). Antioxidant defenses and metabolic 615 depression. The hypothesis of preparation for oxidative stress in land snails. - Comp Biochem 616 Physiol B Biochem Mol Biol. 120: 437-448.

617 Hermes-Lima, M., Moreira, D. C., Rivera-Ingraham, G. A., Giraud-Billoud, M., Genaro618 Mattos, T. C. \& Campos, E. G. (2015). Preparation for oxidative stress under hypoxia and 619 metabolic depression: Revisiting the proposal two decades later. - Free Radic Biol Med. 89: $620 \quad 1122-1143$.

621 Hoelzl, F., Cornils, J. S., Smith, S., Moodley, Y. \& Ruf, T. (2016). Telomere dynamics in 622 free-living edible dormice (Glis glis): the impact of hibernation and food supply. - J Exp Biol. 623 219: 2469-2474.

624 Hudson, N. J. \& Franklin, C. E. (2002). Maintaining muscle mass during extended disuse: 625 Aestivating frogs as a model species. - J Exp Biol. 205: 2297-2303.

626 Kelleher S. R., Silla A. J., Niemelä P. T., Dingemanse N. J. \& Byrne P. G. (2019). Dietary 627 carotenoids affect the development of individual differences and behavioral plasticity. 628 Behav Ecol. arz074.

629 Larcombe, S. D., Tregaskes, C. A., Coffey, J. S., Stevenson, A. E., Alexander, L. \& Arnold, 630 K. E. (2008). The effects of short-term antioxidant supplementation on oxidative stress and 
631 flight performance in adult budgerigars Melopsittacus undulatus. - J Exp Biol. 211: 28596322864.

633 Lichtenthaler, H. K. (1987). Chlorophylls and Carotenoids - Pigments of Photosynthetic

634 Biomembranes. - Methods Enzymol. 148: 350-382.

635 McInerney, E. P., Silla, A. J. \& Byrne, P. G. (2016). The influence of carotenoid 636 supplementation at different life-stages on the foraging performance of the Southern

637 Corroboree frog (Pseudophryne corroboree): A test of the Silver Spoon and Environmental 638 Matching Hypotheses. - Behav Processes. 125: 26-33.

639 McInerney, E. P., Byrne, P. G. \& Silla, A. J. (2017). The effect of dietary antioxidants and 640 exercise training on the escape performance of Southern Corroboree frogs. - Behav

641 Processes. 144: 46-50.

642 Metcalfe, N. B. \& Alonso-Alvarez, C. (2010). Oxidative stress as a life-history constraint: the 643 role of reactive oxygen species in shaping phenotypes from conception to death. - Funct.

644 Ecol. 24: 984-996.

645 Niu, Y., Cao, W., Zhao, Y., Zhai, H., Zhao, Y., Tang, X. \& Chen, Q. (2018). The levels of 646 oxidative stress and antioxidant capacity in hibernating Nanorana parkeri. - Comp Biochem 647 Physiol A Mol Integr Physiol. 219-220: 19-27.

648 Ogilvy, V. \& Preziosi, R. F. (2012). Can carotenoids mediate the potentially harmful effects 649 of ultraviolet light in Silurana (Xenopus) tropicalis larvae? - J Anim Physiol Anim Nutr. 96: $650 \quad 693-699$.

651 Osborne, W. S. (1991). The biology and management of the Corroboree Frog (Pseudophryne 652 corroboree) in NSW. NSW National Parks and Wildlife Service, Hurstville.

653 Oztasan, N., Taysi, S., Gumustekin, K., Altinkaynak, K., Aktas, O., Timur, H., Siktar, E., 654 Keles, S., Akar, S., Akcay, F., Dane, S. \& Gul, M. (2004). Endurance training attenuates 655 exercise-induced oxidative stress in erythrocytes in rat. - Eur J Appl Physiol. 91: 622-627. 
656 Peternelj, T. T. \& Coombes, J. S. (2011). Antioxidant supplementation during exercise

657 training: beneficial or detrimental? - Sports Med. 41: 1043-1069.

658 Powers, S. K., DeRuisseau, K. C., Quindry, J. \& Hamilton, K. L. 2004. Dietary antioxidants 659 and exercise. - J Sports Sci. 22: 81-94.

660 Prokic, M. D., Borkovic-Mitic, S. S., Krizmanic, II, Mutic, J. J., Gavric, J. P., Despotovic, S. 661 G., Gavrilovic, B. R., Radovanovic, T. B., Pavlovic, S. Z. \& Saicic, Z. S. (2017). Oxidative 662 stress parameters in two Pelophylax esculentus complex frogs during pre- and posthibernation: Arousal vs heavy metals. - Comp Biochem Physiol C Toxicol Pharmacol. 202:

$664 \quad 19-25$.

665 Rowe, M., Pierson, K. L. \& McGraw, K. J. (2015). Exploratory behavior is associated with 666 plasma carotenoid accumulation in two congeneric species of waterfowl. - Behav Processes. 667 115: 181-190.

668 Silla, A. J., McInerney, E. P. \& Byrne, P. G. (2016). Dietary carotenoid supplementation 669 improves the escape performance of the southern corroboree frog. - Anim Behav. 112: 213670220.

671 St-Pierre, J. \& Boutilier, R. G. (2001). Aerobic capacity of frog skeletal muscle during 672 hibernation. - Physiol Biochem Zool. 74: 390-397.

673 Svensson, P. A. \& Wong, B. B. M. (2011). Carotenoid-based signals in behavioural ecology: 674 a review. - Behaviour. 148: 131-189.

675 Umbers, K. D. L., Silla, A. J., Bailey, J. A., Shaw, A. K. \& Byrne, P. G. (2016). Dietary 676 carotenoids change the colour of Southern corroboree frogs. - Biol J Linnean Soc, 119: 436677444.

678 Weimerskirch, H., Ancel, A., Caloin, M., Zahariev, A., Spagiari, J., Kersten, M. \& Chastel, 679 O. (2003). Foraging efficiency and adjustment of energy expenditure in a pelagic seabird 680 provisioning its chick. - J Anim Ecol. 72: 500-508. 